\title{
Least Channel Variation Multi-Channel MAC (LCV-MMAC) under Mobile Random Topologies in Mobile Adhoc Networks
}

\author{
Shagufta Henna \\ Bahria University \\ Department of Computer Science \\ Islamabad, Pakistan
}

\author{
Muhammad Bilal Saleemi \\ National Database and Registration Authority \\ Islamabad, Pakistan
}

\begin{abstract}
The use of multiple channels for transmissions has raised several challenges, for example, multi-channel hidden terminal problem, channel switching delay, and control channel saturation problem. Dedicated control channel techniques simplify channel coordination by eliminating the need for synchronization, however the control channel may become the bottleneck for the performance of the network. A better trade off which can solve the coordination problem, and can mitigate the control channel bottleneck is desirable. Least Channel Variant Multi-channel MAC (LCV-MMAC) is a multi-channel MAC based on IEEE 802.11 MAC. The novel part of this protocol is the channel assignment technique, where a mechanism to avoid unnecessary channel assignment and thus channel switching is used. Further, LCV-MMAC avoids channel contention when the control channel is highly saturated. In this paper, we explore the properties of LCV-MMAC through extensive simulations with the help of ns-2, and compare it with popular existing multi-channel MAC protocols in different mobile and static random topologies. Experimental results validate that LCV-MMAC achieves significantly better aggregated throughput, and fairness index than other multi-channel MAC protocols in different random network scenarios.
\end{abstract}

\section{Keywords}

LCV-MMAC, random topologies, performance evaluation of LCVMMAC

\section{INTRODUCTION}

Wireless communication requires access to the wireless medium. Most of the popular MAC protocols [4] called single-channel MAC protocols assume a common shared channel to communicate over the network. IEEE 802.11 MAC is one of the most popular singlechannel based MAC protocols [2]. IEEE 802.11 MAC performs well in single-hop scenarios; however, its performance is detrimental in multi-hop network scenarios due to its CSMA-based approach. Wireless Sensor Networks (WSN) MAC protocols must be capable to operate under different challenges posed by shared access to wireless channel. Examples include collisions caused by the hidden node terminals, and the local contention caused by heavy channel access which degrades the performance of sensor nodes.
Time Division Multiple Access (TDMA) protocols have been used to solve these problems but they are too conservative to handle interference. They assume interference is binary in nature, i.e., it exists or not, however in reality it is probabilistic and is calculated according to the Signal to Interference plus Noise Ratio (SINR) model [10].

It is possible to exploit the channel diversity and capacity of the wireless networks by using multi-channel MAC. Performance of both single-hop and multi-hop wireless networks can be improved by dividing the available bandwidth into multiple channels, and providing access to these channels with the help of multiple access protocols. If a node is allowed to switch over multiple channels, a tremendous increase in throughput is possible immediately. The use of multiple channels reduces the probability of collisions. One more benefit which can be achieved by using multiple channels is the fairness. Due to hidden/exposed node problems in IEEE 802.11 MAC protocols, some flows are at a disadvantage due to topology design resulting in unfairness. Multi-channel MAC can alleviate this unfairness by shifting the disadvantaged flow to a different channel. In IEEE 802.11 devices equipped with half duplex transceivers, it is a challenge to design multi-channel MAC protocols which can fully exploit the channel diversity. In half duplex transceivers, a node is able to transmit or receive at a time. Due to this problem a node is not able to listen on a channel when it is transmitting or receiving on a different channel causing a problem we refer to as multi-channel hidden terminal problem.

Further, in multi-channel MAC protocols three important issues considered are channel assignment, medium access, and channel coordination. Channel assignment is concerned with the selection of channel to be used by a node, while medium access is handling the contention or collisions experienced during a specific channel access. In order to communicate successfully, it is important to negotiate/coordinate the channels effectively to avoid the multichannel hidden terminal problem. If channel assignment and coordination is perfect, the capacity of the networks can be fully exploited.

In [7], we introduce a Least Channel Variation Multiple Channel Medium Access Control (LCV-MMAC) protocol which uses a limited number of channels and a half-duplex transceiver. Frequent exchange of control messages due to frequent channel switching results in control channel saturation problem which builds up queues at the control channel for retransmissions in order to negotiate data 
channels. LCV-MMAC mitigates the control channel saturation and channel switching delay improving the aggregate throughput. LCVMMAC is simple and does not need any network wide periodic synchronization. It avoids frequent channel switching and channel contention, if there is no significant performance gain.

We explore the properties of LCV-MMAC through extensive simulations with the help of ns-2, and compare it with popular existing multiple channel MAC protocols in different random topologies including mobile and static. Experimental results validate that LCV-MMAC outperforms other single channel and multi-channel MAC protocols in different mobile and static random network scenarios. LCV-MMAC achieves better aggregate throughput, latency and fairness index compared to other multi-channel MAC protocols.

\subsection{PERFORMANCE ISSUES IN MULTI-CHANNEL COMMUNICATION}

\section{Multi-Channel Hidden Terminal Problem}

In multi-channel wireless network scenarios, the multi-channel hidden terminal problem is an inborn problem [9]. This problem arises when a node is not able to listen to the handshake procedure on the control channel while it was busy on the data channel for transmission. This node has no information about the channel being reserved by the other nodes during the handshake procedure and therefore may inadvertently select the same data channel for the next transmission, thus causing a collision. Dynamic Channel Assignment (DCA) [25] solves the multi-channel hidden terminal problem by using two transceivers, one of which is permanently tuned to the control channel and a node is able to listen on the control and data channel simultaneously, however this solution is expensive. Multi-channel MAC (MMAC) [16] solves this problem by using a split phase approach where handshake occurs during the control phase, and therefore neighboring nodes have information about the channel usage. Another possible solution to reduce the multi-channel hidden terminal problem is careful channel selection.

\section{Missing Receiver Problem or Deafness}

Another common problem in multi-channel MAC protocols is the missing receiver or deafness problem, which occurs when a receiver is not able to receive the control packets because it was busy in transmission on some other channel and therefore is not able to respond. Missing receiver problem persists in the protocols, where a dedicated control channel is used to exchange the control messages. This problem induces an increased number of retries resulting in congestion, delay and unfairness [6]. DCA [25] avoids this problem by using multiple transceivers, one of which is always tuned to the control channel, and therefore the receiver cannot miss any control packet. MMAC [16] uses the control phase to exchange any channel information, and therefore the receiver cannot miss the channel information.

\section{Channel Switching Delay Problem}

Multi-channel MAC methods based on dynamic channel assignment require unnecessary switching of the radio on different channels without any performance gains, which adds delay, and increases the overhead of channel negotiation and coordination. In IEEE $802.11 \mathrm{~b}$, the time required to switch from one channel to another is $224 \mu s$ [1], and in Chipcon $C C 2420$ radio it is $300 \mu s$ [3]. Control Channel Saturation Problem

In dedicated control channel based MAC approaches, the control channel has significant impact on the aggregated throughput of the data channels. Compared to the three other categories of multichannel MAC protocols, i.e., split phase, frequency hopping, and multiple rendezvous, dedicated control channel based approaches do not need any synchronization and therefore are simple to implement. However, one of the drawbacks of such approaches is the control channel saturation problem due to channel negotiations. This may result in underutilization of data channels. This problem has been discussed in detail in [14] and [22]. DCA [25] is an example of a multi-channel MAC protocol which is prone to the control channel saturation problem. Certain dedicated control channel approaches based on the IEEE 802.11 MAC standard have been proposed with improved performance. In [26], the original MAC design has been improved in terms of energy efficiency, however the control channel saturation problem has not been addressed. In [5], an approach is proposed to reduce the underutilization of data channels due to the control channel reservation process. This scheme improves the aggregated throughput of data channels when the control channel is not saturated.

\section{RELATED WORK}

In this section, we review some existing multi-channel MAC protocols which suffer from the control channel saturation problem. Vaidya and Jungmin [16] propose Multi-channel MAC (MMAC) which is a well known time multiplexed (split phase) multi- channel MAC protocol. MMAC periodically transmits beacons with a beacon period of $100 \mathrm{~ms}$, which is further subdivided into an Ad hoc Traffic Indication Message (ATIM) window of $20 \mathrm{~ms}$ and a DATA window of $80 \mathrm{~ms}$. MMAC exploits ATIM windows of IEEE 802.11's Power Saving Mechanism (PSM) and extends it for channel reservations. During the ATIM window, all nodes listen on the common control channel and contend for the free channels. After channel reservation, data transmissions take place on all the available data channels during the DATA window. For data transmission, both sender and receiver switch to the reserved channel. In order to handle the hidden terminal problem, MMAC also uses RTS, CTS, and Reservation (RES) packets to negotiate the data channels during the ATIM window. These messages also help to update the information about the reserved channels. Although MMAC requires only one transceiver at each node, the use of synchronizing beacons adds complexity in its implementation. Further, periodic exchange of RTS/CTS, ATIM, ATIM Acknowledgement (ATIM-ACK), and ATIM Reservation (ATIM-RES) packets during the ATIM window negatively impact the efficiency of the control channel resulting in reduced scheduling capacity. In MMAC, during highly congestive scenarios, how to solve the control channel saturation problem remains open.

DCA [24] is a multi-radio multi-channel MAC protocol and requires two radio interfaces. One interface is permanently tuned to the control channel to facilitate the channel negotiation process. The second interface is able to dynamically switch to a reserved data channel to transmit or receive data. In DCA, access to the control channel is similar to IEEE 802.11 MAC. The use of a dedicated control channel eliminates the need for synchronization. Further, the RTS/CTS mechanism on the control channel tuned to the dedicated radio interface makes DCA more immune to the hidden terminal problem. However, one of its major limitations is the control channel saturation, where the control channel may become a bottleneck for the network performance. The right bandwidth for the control channel is tightly coupled with the traffic; a wide control channel results in bandwidth wastage and a narrow channel may become a bottleneck to network performance. This control channel saturation problem has been discussed in [23]. Further, DCA requires an extra radio interface and therefore is an expensive solution. 
Asynchronous Multi-channel Coordination Protocol (AMCP) 9] uses a dedicated control channel and $n$ data channels. Nodes exchange control messages on the control channel in order to negotiate and reserve the data channel. Each node is equipped with a half duplex transceiver where a node can transmit or receive at a time. Each node maintains a table, where each table entry records a channel, a bit indicating its availability and a timer indicating the duration of the time a channel is being used by other neighbouring nodes. Further, AMCP uses a prefer variable to decrease the probability of collisions which in turn increases the aggregated throughput and fairness among the flows. Nodes in AMCP defer the channel availability for a duration equal to Distributed Inter-frame Spacing (DIFS) plus channel switching duration which negatively impacts the capacity of the control channel. The DIFS is the amount of time a node must wait for a clear channel before starting a new transmission. High contention or queues at the control channel may result in underutilization of data channels.

Bi-directional Multi-channel MAC protocol (Bi-MMAC) $[11]$ is an extension to MMAC with bidirectional flow of data. Bi-MMAC uses the RTS/CTS handshake mechanism complemented with Channel Reservation Notification (CRN). A sender explicitly sends $\mathrm{CRN}$ to its neighbours about the channel reservation and its duration. Similar to MMAC, Bi-MMAC uses $K$ channels where one channel is used as the control, and the remaining $K-1$ as the data channels. However, Bi-MMAC differs from other multi-channel MAC protocols by facilitating the DATA frame exchange in both directions. In particular Bi-MMAC improves the performance of TCP, where both the DATA and ACK packets traverse in opposite directions in a network. The Bi-MMAC protocol is named as bidirectional as the receiver may append any data to the acknowledgement and send it back to the sender. This bidirectional flow of data saves the handshake procedure for sending data from receiver to sender. Specifically, this approach improves the performance of TCP transfers, where TCP DATA and TCP ACKs packets traverse in opposite directions. However, the exchange of RTS/CTS/CRN on the control channel may result in a bottleneck in heavily loaded network scenarios and can adversely affect the performance of BiMMAC.

Receiver Directed Transmission (RDT) [15] is one of the popular works for dynamic channel selection. In RDT, each node is equipped with a half duplex transceiver and each node assigns itself a quiescent channel to which a node stays tuned whenever it is idle. In order to transmit data to a receiver, the transmitter must switch its interface to the quiescent channel of the receiver. The rest of the transmission mechanism is similar to the 802.11 MAC protocol with RTS/CTS enabled. After the successful data transmission,the transmitter tunes back to its quiescent channel. RDT assumes a separate mechanism for the assignment of the quiescent channel, and for the distribution of selected quiescent channel information to the neighboring nodes. These mechanism can be implemented by using a cross layer approach. In highly contended network scenarios, the performance of RDT may be even poorer compared to single channel IEEE 802.11 MAC due to deafness and hidden node terminal problems. Extended Receiver Directed Transmission (xRDT) protocol [13] extends RDT, in which different nodes can use possibly different quiescent channels complemented with a busy tone radio to inform the neighboring nodes about the ongoing reception. The additional busy tone radio mitigates the multi-channel hidden node and deafness problems inherent to RDT. However, the need for an extra radio adds cost and complexity in its implementation. Further, this scheme results in wastage of bandwidth for the control channel used to send busy signals only.
Local Coordination-based Multichannel MAC (LCM-MAC) [13] needs a single interface compared to XRDT, and provides multichannel support by using coordinated channel negotiation and channel switching. Local coordination in LCM-MAC helps to schedule transmissions without the need for any time synchronization. The transmission schedule of LCM-MAC consists of two phases: control window and data window. During the control window phase, all nodes are tuned to the same channel to transmit or receive control packets. In contrast to xRDT, nodes use a common channel to negotiate and reserve the channel during the control window. This common channel also acts as a data channel during the data window. Access to the control channel is similar to 802.11 MAC. After negotiating the channel during the control window, nodes use the reserved channel for transmission during the data window. After transmission the nodes switch back to the default channel to listen/negotiate any channel reservations. Additional signaling packets for channel reservation during the control phase increase the signaling payload similar to [16] resulting in wastage of bandwidth for the control, and data channel.

The multi-channel MAC approach proposed in [4] employs a dedicated control channel. Similar to LCM-MAC, the control channel in this approach acts both as a signaling as well as data channel. The protocol works in two phases: Contention Free Interval (CFI) and Contention Reservation Interval (CRI). During the CRI, nodes contend for the data channel and defer their transmission until the start of CFI. The deferment of the transmission results in underutilization of data channels and also degrades the capacity of the control channel.

Several other multi-channel MAC protocols including receiver initiated channel hopping with dual polling [20, 19, 18] and Hop Reservation Multiple Access (HRMA) [17] are well known frequency hopping spread spectrum techniques and are worth to mention here. $|20|$ is based on a dedicated control channel approach where nodes listen to the control channel in order to synchronize their hopping sequence. Frequent channel hopping results in longer channel switching delays degrading the performance of the protocol.

Other popular techniques include multi-radio multi-channel MAC approaches [21, 8, 12, 15, 12] with static and dynamic channel selection and target improved network throughput by reducing the number of collisions. The multi-channel approach in [8] implements $N$ data channels and a dedicated control channel. The RTS/CTS used in this scheme is not sufficient to cope with the hidden node problem and therefore triggers numerous retransmissions degrading the capacity of the control channel. This technique assumes that a node is able to sense and receive on all the channels simultaneously, which incurs high overhead cost due to frequent channel switching.

In this paper, we evaluate the performance of a multi-channel MAC protocol called LCV-MMAC which is based on the dedicated control channel in different random mobile and static network topologies.

\section{PROTOCOL DESCRIPTION OF LCV-MMAC}

In LCV-MMAC, each node is node is equipped with a half-duplex transceiver. In LCV-MMAC, each node maintains a channel table including the channel,a timer when channel will be released,and a bit indicating the availability of the channel. In LCV-MMAC, each node detects the control channel saturation by using the channel busyness ratio $R_{\text {busy }}$ as discussed below. In no control channel saturation is detected, node continues with the last known data channel without any channel switching. If no channel is known, node looks 
for an available channel with minimum usage. Channel selection is not needed for every transmission. If a neighbour already knows the receiver's channel, it selects the same channel for transmission. Further, every node detects the control channel saturation by using the channel busyness ratio $R_{\text {busy }}$ [27]. $R_{\text {busy }}$ indicates a sign of control channel saturation. Given $T_{s u c}$, and $T_{c o l}$ be the time periods associated with the successful transmission, and a transmission resulting in collision, respectively. Then with the RTS/CTS enabled [27:

$$
\begin{gathered}
T_{s u c}=r t s+c c t s+c r n+d a t a+a c k+3 s i f s+d i f s \\
T_{c o l}=r t s+c t s \_t i m e o u t+d i f s=r t s+\text { eifs }
\end{gathered}
$$

Where rts denotes time to send an RTS, and ccts denotes time to receive a successful CTS packet from the receiver. $\mathrm{crn}$ in Equation 1 denotes the time to send a control channel reservation notification to neighbouring nodes about the channel reservation. The data denotes the average length of the data packet in seconds for successful transmission. sifs, and difs denote short inter-frame spacing, and distributed inter-frame spacing. When a node experiences a collision it adjusts its NAV with an extended inter-frame spacing eifs period as shown in Equation 2 The channel busyness ratio $R_{\text {busy }}$ of the control channel can be computed as follows:

$$
R_{b u s y}=\frac{T_{s u c}+T_{c o l}}{T_{t o t}}
$$

Equation 3 defines the $R_{b u s y}$ as the ratio of the total lengths of busy periods due to collisions or successful transmissions to the total time $T_{\text {tot }}$ during a time interval. If the control channel is saturated as indicated by the $R_{\text {busy }}$, a node continues with the last known data channel to transmit to the receiver without any channel switching. It is likely that the receiver stays switched on the same channel. If no control channel saturation is detected, node contends the control channel for to negotiate the channel with lower usage. Once a channel has been reserved between the sender and receiver, DATA is transferred by using it. All neighbouring nodes overhearing the channel negotiation defer their data transmission according to the duration of the transmission specified during the channel negotiation process.

\section{PERFORMANCE EVALUATION}

We evaluate the performance of LCV-MMAC with the help of extensive simulations by using ns- 2 . We compare the performance of LCV-MMAC with MMAC, AMCP, DCA, and single channel 802.11 in different static and mobile network topologies. DCA is a well known representative of multi-channel MAC protocols which suffer from the control channel saturation problem. MMAC is a representative of multi-channel MAC protocols based on the splitphase approach, and finally we also compare the performance of LCV-MMAC with single channel standard 802.11 MAC. We run all experiments for a simulation time of 300 seconds. For all the experiments we have used the AODV routing protocol. Unless stated otherwise, the distance between the nodes is $250 \mathrm{~m}$. We have used 4 channels for LCV-MMAC, 1 for the control and the other 3 as the data channels. Other IEEE 802.11 simulation parameters are listed in the Table 4.1

\subsection{Static Random Topology Vs. Mobile Random Topology}

In order to analyse the effect of mobility on multi-channel protocols we have deployed 100 nodes in a simulation area of $1000 \mathrm{~m} \times$ $1000 \mathrm{~m}$. We have compared the performance of LCV-MMAC with other multi-channel protocols in both static and mobile network topologies. Further, we simulate the mobility of the nodes by using the random way point mobility model, where nodes can move with a maximum speed of $10 \mathrm{~m} / \mathrm{s}$ with $0 s$ pause time. We varied the seed for both random topology generator and the random source/destination. For 10 random TCP connections, we tested 10 random topologies yielding a total of $10 \times 10=100$ topologies/scenarios. Each simulation run lasts for 300 seconds.

LCV-MMAC outperforms 802.11 MAC and all other multi-channel protocols in both static and mobile random topologies. We demonstrate the mobility in the network which makes the situation somewhat more challenging for channel access and therefore use of multiple channels. In a network with mobility, a single flow may traverse through multiple routes throughout the simulation and therefore may choose the best route at different intervals offering different degrees of channel diversity. The full benefit of multiple channels can be realized when there is an increase in the number of flows in the network. LCV-MMAC leverages this benefit better compared to other multi-channel protocols, and therefore demonstrates significant performance improvement over 802.11 MAC, and other multi-channel protocols. In a random network due to dynamics of control channel contention LCV-MMAC mitigates control channel saturation and effectively utilizes the data and control channels.

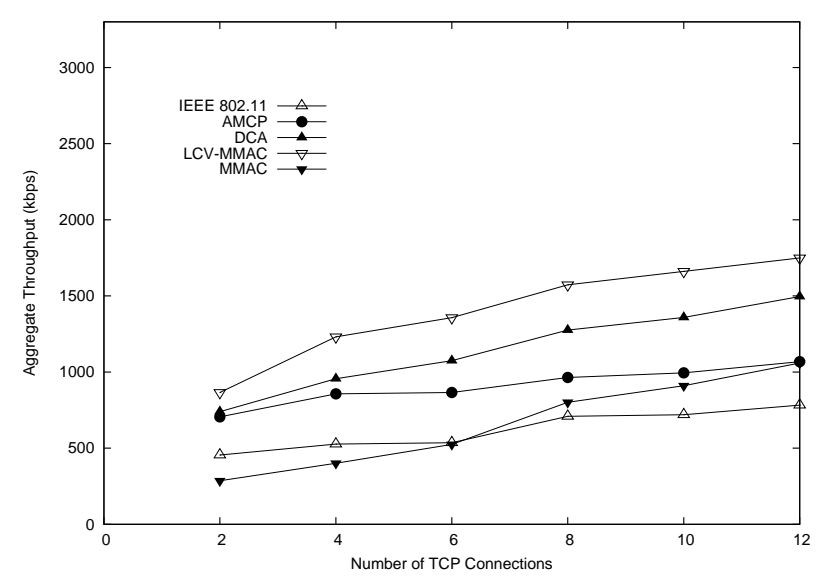

Fig. 1. Aggregated throughput versus. No. of connections in static toplogy

As shown in Figure 1 and Figure 4 the aggregated throughput of MMAC for both static and mobile topologies is poor compared to other multi-channel protocols due to the need of synchronization. In multi hop scenarios, it is very difficult to achieve synchronization as only nodes which are involved in communication can exchange ATIM messages during the ATIM window. Mobility in a network results in increased route discoveries which increase the packet delay. Multi-channel diversity in mobile networks mitigates this effect and the use of extra channels always results in lower delays. From 


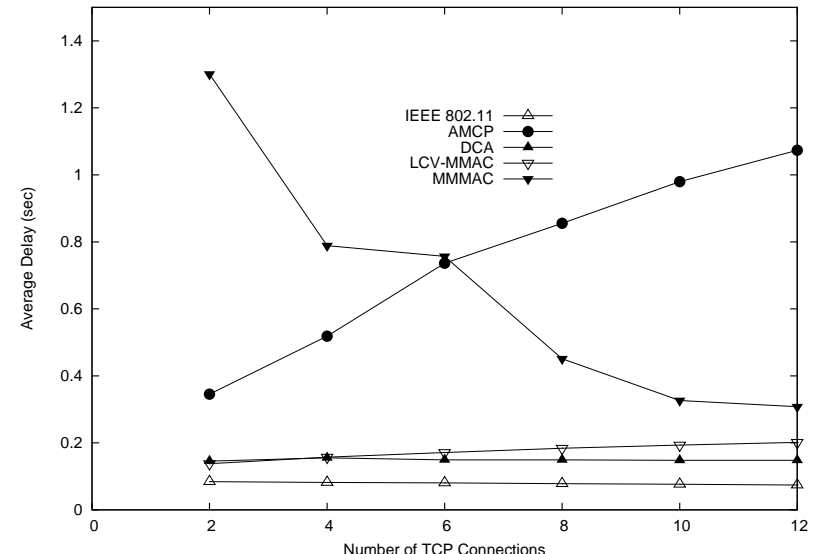

Fig. 2. Average delay versus No. of connections in static toplogy

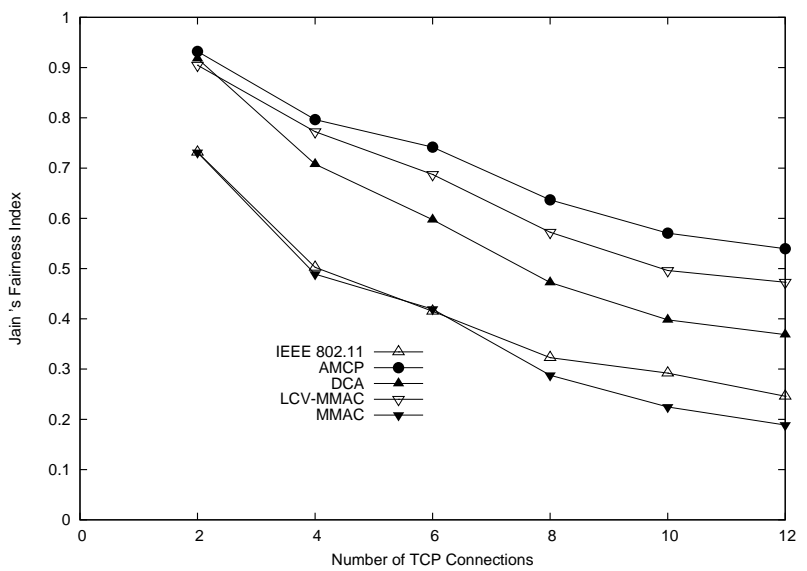

Fig. 3. Fairness index versus No. of connections in static toplogy

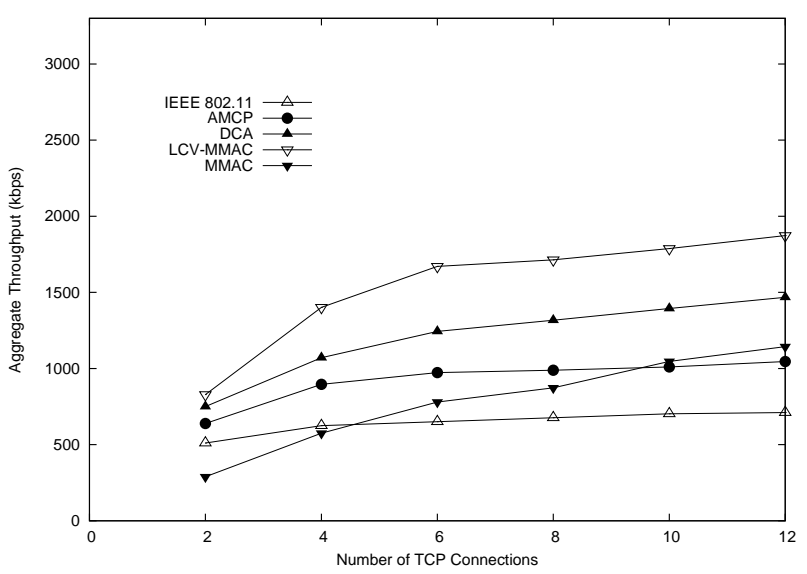

Fig. 4. Aggregated throughput versus. No. of connections in mobile toplogy

the Figure 2 and Figure 5 it is clear that most of the multi-channel protocols have almost the same delay performance in both the static

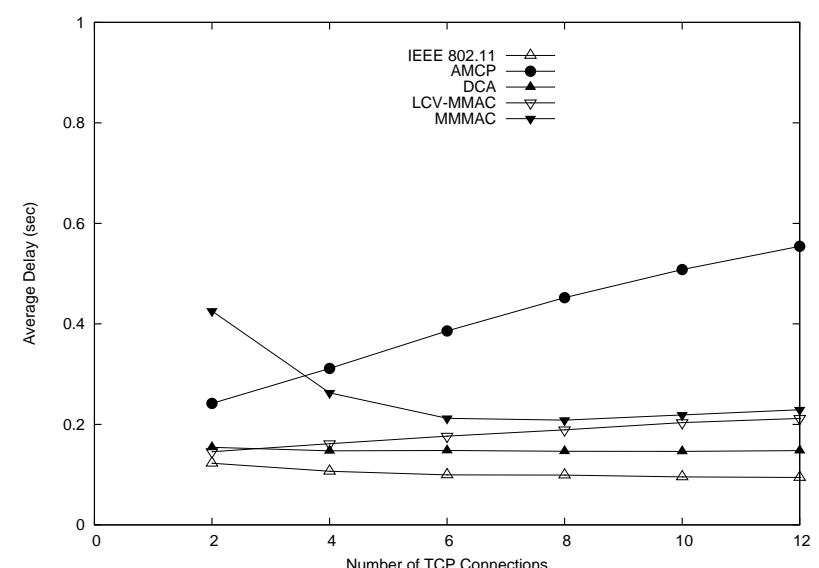

Fig. 5. Average delay versus No. of connections in mobile toplogy

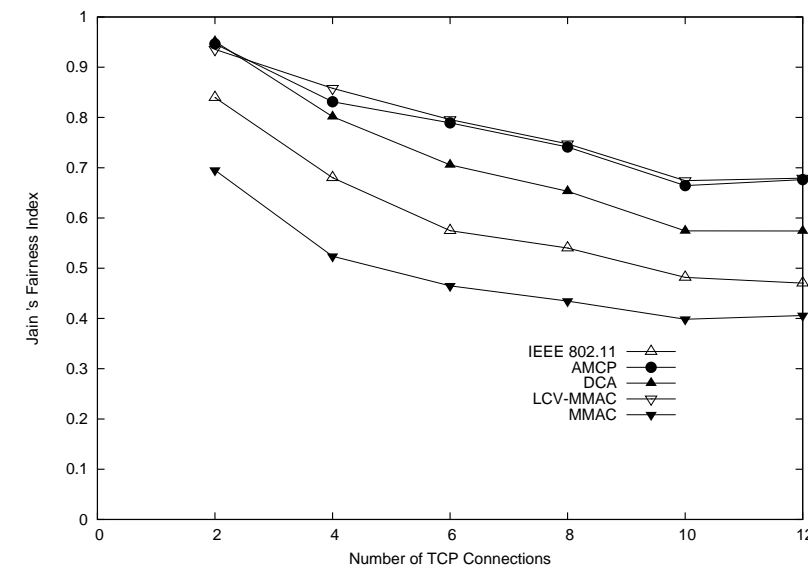

Fig. 6. Fairness index versus No. of connections in mobile toplogy

\begin{tabular}{ll}
\hline Parameter Name & Parameter Value \\
\hline SIFS & $10 \mu \mathrm{s}$ \\
DIFS & $50 \mu \mathrm{s}$ \\
Long Retry Limit & 7 \\
Short Retry Limit & 4 \\
$C W_{\min }$ & 31 \\
$C W_{\max }$ & 1023 \\
EIFS & $364 \mu \mathrm{s}$ \\
DATA packet & $8000 \mathrm{bits}$ \\
Bit rate for DATA packets & $2 \mathrm{Mbps}$ \\
MMAC ATIM window & $20 \mathrm{~ms}$ \\
MMAC Beacon interval & $100 \mathrm{~ms}$ \\
Channel switching delay & $224 \mu \mathrm{s}$ \\
RTS packet & 160 bits + Phy header + MAC \\
& header \\
CCTS/RCTS,ACK packet & 112 bits + Phy header \\
CRN packet & 160 bits + Phy header + MAC \\
& header \\
\hline
\end{tabular}

IEEE 802.11 system parameters

and mobile networks. However, the delay experienced by MMAC significantly reduces in mobile networks. 
In both the static and mobile random networks, Jain's fairness index as shown in Figure 3 and Figure 6 reduces for almost all the MAC protocols due to increased competition among the flows. LCV-MMAC has better fairness compared with other MAC protocols for all the traffic loads.

\section{CONCLUSION}

The use of multi-channel MAC protocols improves the performance of wireless networks, in particular throughput and fairness. The main objective of most of the research in multi-channel MAC protocols is to find out ways which can use multiple channels in an efficient way, thus further improving the aggregated throughput and fairness.LCV-MMAC improves the aggregated throughput in different network scenarios including random topologies compared to single channel and some other multi-channel MAC protocols. Results obtained by conducting experiments reveal that LCVMMAC demonstrates significantly better aggregated throughput performance compared to MMAC and AMCP in different mobile and static random network scenarios.

\section{REFERENCES}

[1] Jardosh Ami, Ramachandran Krishna, Almeroth Kevin, and Belding-Royer Elizabeth. Understanding congestion in IEEE 802.11 b wireless networks. In Proc. of the ACM SIGCOMM conference on Internet Measurement, pages 279-292, 2005.

[2] F. Cali, M. Conti, and E. Gregori. IEEE 802.11 wireless LANS: capacity analysis and protocol enhancement. pages 142-149, 1998.

[3] Chipcon Products. 2.4 GHz IEEE 802.15.4/zigbeed-ready RF transceiver.

[4] I. Demirkol, C. Ersoy, and F. Alagoz. MAC protocols for wireless sensor networks: A survey. IEEE Communications Magazine, 44(4):115-121, 2006.

[5] M. D. Felice, G. Zhu, and L. Bononi. Future channel reservation medium access control (FCRMAC) protocol for multiradio multi-channel wireless mesh networks. In Proc. of the ACM Symposium on Communications and Information Technologies, pages 71-79, 2008.

[6] B. S. V. Gambiroza and E. Knightly. End-to-end performance and fairness in multihop wireless backhaul networks. pages 287-301, 2004.

[7] Shagufta Henna and Thomas Erlebach. Least channel variation multi-channel mac (LCV-MMAC). In Proc. of the 12th International Conference on Ad Hoc Networks and Wireless, 2013.

[8] N. Jain, S. Das, and A. Nasipuri. A multichannel CSMA MAC protocol with receiver-based channel selection for multihop wireless networks. pages 432-439, 2001.

[9] S. Jingpu, T. Salonidis, and E.W. Knightly. Starvation mitigation through multi-channel coordination in CSMA multi-hop wireless networks. pages 214-225, 2006.

[10] Dimitios Koutsonikolas, Theodoros Salonidis, Henrik Lundgren, Pascal LeGuyadec, Y. Charlie Hu, and Irfan Sheriff. TDM MAC protocol design and implementation for wireless mesh networks. In Proc. of the Int'l Conf. on Context Aware Systems and Applications, pages 1-28, 2008.

[11] T. Kuang and C. Williamson. A bidirectional multi-channel MAC protocol for improving TCP performance on multihop wireless ad hoc networks. pages 301-310, 2004.
[12] J. Li, Z. Haas, M. Sheng, and Y. Chen. Performance evaluation of modified IEEE 802.11 MAC for multi-channel multihop ad hoc network. In Intl. Conf. on Advanced Information Networking and Applications, pages 312-317, 2003.

[13] R. Maheshwari, H. Gupta, and S. Das. Multichannel MAC protocols for wireless networks. In IEEE Communication Society Conference on Sensor, Mesh and Ad Hoc Communications and Networks, pages 25-28, 2006.

[14] J. Mo, H. Sheung, W. So, and J. Walrand. Comparison of multichannel MAC protocols. pages 209-218, 2005.

[15] Shacham and P. King. Architectures and performance of multichannel multihop packet radio networks. IEEE Journal on Selected Areas of Communication, 5(1):1013-1025, 1987.

[16] J. So and N. Vaidya. Multi-channel MAC for ad hoc networks: Handling multi-channel hidden terminals using a single transceiver. pages 222-233, 2004.

[17] Tang and J. J. Garcia-Luna-Aceves. Hop-reservation multiple access (HRMA) for multichannel packet radio networks. pages 388-395, 1998.

[18] A. Tzamaloukas and J. Garcia-Luna-Aceves. Channelhopping multiple access with packet trains for ad hoc networks,. In IEEE International Conference on Device Multimedia Communications, 2000.

[19] A. Tzamaloukas and J. J. Garcia-Luna-Aceves. Channelhopping multiple access. In IEEE International Conference on Communications, pages 415-419, 2000.

[20] A. Tzamaloukas and J. J. Garcia-Luna-Aceves. A receiverinitiated collision-avoidance protocol for multi-channel networks. pages 189-198, 2001.

[21] N. Vaidya and P. Kyasanur. Routing and interface assignment in multi-channel multi-interface wireless networks. pages 2051-2056, 2005.

[22] J. C.P. Wang, M. Abolhasan, F. Safaei, and D. Franklin. A survey on control separation techniques in multi-radio multichannel MAC protocols. In Proc. of the Symposium on Communications and Information Technologies, pages 854-859, 2007.

[23] Peng-Jung Wu and Chung-Nan Lee. Connection-oriented multi-channel MAC protocol for ad-hoc networks. Computer Communications, 32(1):169-178, 2009.

[24] S. Wu, Chih-Yu Lin, Yu-Chee Tseng, and Jang-Ping Sheu. Multi-channel MAC for ad hoc networks: Handling multichannel hidden terminals using a single transceiver. pages 232-237, 2000.

[25] Shih-Lin Wu, Chih-Yu Lin, Yu-Chee Tseng, and Jang-Ping Sheu. A new multi-channel MAC protocol with on demand channel assignment for mobile ad-hoc networks. pages 232237, 2000.

[26] Shih-Lin Wu, Chih-Yu Lin, Yu-Chee Tseng, and Jang-Ping Sheu. A multi-channel MAC protocol with power control for multi-hop mobile ad-hoc networks. The Computer Journal, 45(1):101-110, 2002.

[27] Shongqiang Zhai, Xiang Chen, and Yuguang Fang. Call admission and rate control scheme for multimedia support over IEEE 802.11 wireless LANs. Wireless Networks, 12(4):451463, 2006. 